\title{
Reflections on War Ethics in the Employment of UAVs
}

\section{Zhaolei GUO' ${ }^{1}$ ' Yiping ZHENG $^{2}$}

Center for International Strategy and Security, Nanjing University of Aeronautics and Astronautics, China

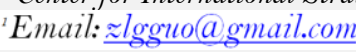

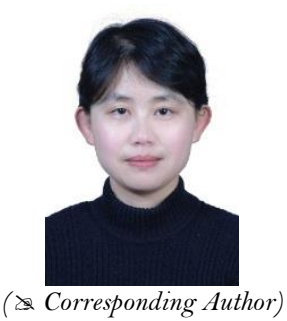

\begin{abstract}
The debate over the ethical justification of Unmanned Aerial Vehicles has been growing heated for years with the proliferation use of UAVs. At the center of this debate are the underlying question of war ethics and the concerns of whether UAVs will be able to fully comply with war ethics in future. This article is trying to articulate the relevant precepts and points out some misunderstanding clouding UAVs. After focusing the moral debates based on the basic principles of the just war, this article contends that regulations should respond to the character that UAVs is in incremental development to adjust themselves, rather than ban simply.
\end{abstract}

Keywords: UAVs, Autonomous weapon, Just war, Distinction, Proportionality.

Citation | Zhaolei GUO; Yiping ZHENG (2018). Reflections on War Ethics in the Employment of UAVs. Asian Journal of Social Sciences and Management Studies, 5(3): 131-136.

History:

Received. o June 9018

Revised: 7 August 2018

Accepted: 5 September 2018

Published: 27 September 2018

Licensed: This work is licensed under a Creative Commons

Attribution 3.0 License (cc) I

Publisher:Asian Online Journal Publishing Group
Contribution/Acknowledgement: Both authors contributed to the conception and design of the study.

Funding: The research is supported by "the Fundamental Research Funds for the Central Universities" NO. NJ20140035 Competing Interests: The authors declare that they have no conflict of interests.

Transparency: The authors confirm that the manuscript is an honest, accurate, and transparent account of the study was reported; that no vital features of the study have been omitted; and that any discrepancies from the study as planned have been explained.

Ethical: This study follows all ethical practices during writing.

\section{Contents}

1. Introduction 


\section{Introduction}

In recent years, with the rapid rise in the employment of UAVs and consequent damage to noncombatant, scholars and international organizations, such as Human Rights Watch, the International Committee of the Red Cross (ICRC) has been shown continuous concern over the legal and moral deployment of UAVs in the conflicts.

According to attitudes towards UAVs, scholars are divided on the extent to which UAVs are moral and legal. their views spread just like a spectrum, at the one end, some assert that UAVs is unlawful and should be forbidden as soon as possible; at the other side, some claim that UAV not only satisfy all current law and ethical codes, it is also helpful to improve the efficiency. However, most of the scholars spread in the middle range of the spectrum, whether the deployment of UAVs is lawful or ethical depend on the specific circumstances.

Those who think UAVs should be forbidden as soon as possible hold two strands of arguments. One strand of views think UAVs has violated the law or ethical principle, so we should forbid the employment and development of UAVs. Towards the argument, the paper will deal with it as below. However, for the other strand, what they actually object to is lethal autonomous weapons, rather than UAVs. The opponents assert that the employment of UAVs is on the slippery slope towards lethal autonomous weapons and autonomous weapons are morally impermissible, so the development of the UAVs should be forbidden. The moral permissibility of lethal autonomous weapons is beyond the scope of my paper. We will point out that some objections to UAV are out of the fear of the future imaginable horrible scene, or even are caused by misleading perceptions. In this article, we argue that in many cases grave moral reflections of UAVs are distracted or weakened by ambiguous concept defines.

This paper seeks to outline the current prevailing misunderstanding of the use of UAVs. And examines debates pertaining to the distinction and proportionality of the deployment of UAVs based on the just war theory.

The paper is split into five areas:

In the second section, considering criticism of UAVs is partly due to the misconceived conception of UAVs, which arouse many irrelevant discussion distracting our precious attention, we frame the definition of UAVs and debunk some arguments misleading public debate, before going to the discussion about ethical concerns in the case of UAVs.

In the third section, we enter into the discussion of moral concerns surrounding UAVs underpinned by the just war theory. In the fourth section, based on the above discussion, we make suggestions to improve moral performance of UAVs. Finally, we claim that to prompt UAVs to satisfy moral norm, it is imperative to clarify the ambiguous text, make detailed guidelines more practical, improve transparency and adopt appropriate modification adapting to changes.

\subsection{Misunderstanding about UAVs}

\subsubsection{The Definition of UAVs}

In the paper, we are referencing UAVs as a weapon system commonly known as 'Unmanned Aerial Vehicles', a kind of autonomous weapons systems, but not fully autonomous. It has several other names, drones, robots or autonomous weapon system. They are colloquial and ambiguous. Therefore, when the above-mentioned terms appear in a paper, they either refer to the same thing we are discussing or the other way around. The ambiguity of the definition is easier to arouse debates. More importantly, without accurate definition, there are greater possibilities that discussions do not occur in the same field.

To avoid misunderstanding and unnecessary debates, we need to first classify the various level of the autonomous weapon system. According to the U.S. Department of Defense's definition, an autonomous weapon system means: "a weapon system that, once activated, can select and engage targets without further intervention by a human operator. This includes human-supervised autonomous weapon systems that are designed to allow human operators to override operation of the weapon system, but can select and engage targets without further human input after activation." "In addition, U.K. Ministry of Defense identifies an autonomous system to be' capable of deciding a course of action, from a number of alternatives, without depending on human oversight and control, although these may still be present (MoD, 2011).

The crux of the matter in both definitions is that a system has the ability of making decision without human interface. However, to what extent the system can decide by itself, both definitions have grey areas. It is one of the reasons for the heated debates.

According to different levels of autonomy, U.S Department of Defense classifies lethal unmanned weapon systems into three categories: that is, semi-autonomous systems, also called launch and leave weapons; humansupervised autonomous system and fully autonomous weapon system. Correspondingly, Human Rights Watch call them respectively: a human in the hoop; a human on the hoop. Basically, one-to-one correspondence. The classification reiteration is primarily based on the relationship between the human operator and the system operation. We list them in the following Table.

Table-1. Classification of the autonomous weapon system

\begin{tabular}{l|l|l|l}
\hline Classification by DOD & $\begin{array}{l}\text { Classification by Human } \\
\text { Rights Watch }\end{array}$ & Examples \\
\hline $\begin{array}{l}\text { Semi-autonomous weapon system } \\
\text { (launch and leave weapons) }\end{array}$ & A human in the loop & $\begin{array}{l}\text { A human to direct the } \\
\text { system to select a target } \\
\text { and attack it }\end{array}$ & $\begin{array}{l}\text { AGM-130,AGM- } \\
65\end{array}$ \\
$\begin{array}{l}\text { The human-supervised } \\
\text { autonomous weapon system }\end{array}$ & A human on the loop & $\begin{array}{l}\text { Under human operator } \\
\text { oversight }\end{array}$ & $\begin{array}{l}\text { U.S.: the Aegis at } \\
\text { sea/ the Patriot on } \\
\text { land; } \\
\text { Israel: Iron Dome }\end{array}$ \\
$\begin{array}{l}\text { The fully autonomous weapon } \\
\text { system }\end{array}$ & A human out of the loop & $\begin{array}{l}\text { Without any human } \\
\text { interface }\end{array}$ & hemon \\
\hline
\end{tabular}

' DEP’T OF DEF., DIRECTIVE 3000.09, AUTONOMY IN WEAPON SYSTEMS 13-14 (Nov. 2, 2012). 
Based on the above discussion, we can infer that the semi-autonomous weapon system is a commonplace of contemporary warfare and the human-supervised autonomous weapon system is in operation, but a fully autonomous weapon system is still in the future. Therefore, what we discuss is semi-autonomous weapon system and human-supervised autonomous weapon system, rather than a fully autonomous weapon system.

\subsection{Distinction between Autonomous and Automate}

Some of the continuing moral debates over UAVs obscure the distinction between autonomous and automate. Current applied artificial intelligence is still based on the binary IF/THEN statement, which means that the machine decision is still based on the limited and certain information, far short of human decision based on uncertainty. Actually, just as Peter Lee said, the current autonomous weapons falls far short of the criterion of autonomy, precisely, should be called 'pseudo-autonomy', 'a high level of automation'. Lee (2018) fully autonomy has not yet come into reality and the UAVs we are now discussing are a far cry from the fully autonomous weapon. Therefore, the worry that the autonomy of the machine will take a human out of the firing loop is suffering from imaginary fears. In reality, many criticisms over UAVs in operation is leveling at the deployment of a military weapon of AI technology, rather than UAVs. This is not actually an objection specific to UAVs in principle but rather aiming to AI. Some believe that perfect mix of AI and the lethal autonomous weapon will produce a fully lethal autonomous weapon, which will achieve or even surpass human autonomy. Those taking a sanguine view think it will benefit the entire population, while the pessimistic view thinks that 'spell the end of the human race'. The discussion over the issue is entered into the 'Terminator conundrum', Kiggins (2018) which far exceeds the scope of our paper.

Coming back to the current context, the worry seems to fall short of logic. The worry is a sentiment, but not rather a coherent and compelling ethical argument. The logic reasoning of those who persist on thoroughly ban UAVs is that we should now forego taking morally correct action (protect our warriors as best we can to avoid unnecessary harm to them) to prevent ourselves from acting unjustly in the future. The moral reasoning seems odd. Just like Strawser said it is like that 'we should do something wrong now to better stop ourselves from doing something wrong in the future' (Strawser, 2010).

\subsection{Policymakers or UAVs?}

In addition, the bad reputation of UAV partly caused by the utilization in some military strike actions whose morally justification itself is in dispute. It is wrong or even illegal or unmoral military decisions, rather than UAV caused excessive losses to civilians and civilian facilities. Therefore, UAVs should not take full responsibility for the harm caused by these attacks and the incurred criticism thereby. Some criticism leveling at UAVs are making a scapegoat for moral disasters, which will disguise some deep problems. It is unhelpful to solve the moral problems and even distract attention from constructive discussions. If it is the wrong military decision that caused excessive collateral damage, who should take the responsibility, the policymakers who make the decision or UAVs employed by decisions?

As the saying goes, "A sword is never a killer, it is a tool in the killer's hands." Certain munitions can be used in some operations to violate the law and ethical rule, but in other circumstances, the same munitions may be used to protect and innocent civilians justice warriors. The crux of the problem is that how the man holding the sword use the munition. It is unfair to make UAVs the scapegoat for the immoral action of policymakers.

\subsection{Debates about the Principles of Just War Theory}

After the definition of the issue and clarification of the misunderstanding, the following analysis focuses on the moral concerns revolving around principles of distinction and proportionality as the centerpiece of the just war theory.

\section{The Principle of Distinction}

Distinction is reflected in several rules, the most invoked code is Article 48 of the Protocol Additional to the Geneva Convention, which prescribes that "In order to ensure respect for and protection of the civilian population and civilian objects, the Parties to the conflict shall at all times distinguish between the civilian population and combatants and between civilian objects and military objectives and accordingly shall direct their operations only against military objectives."

\subsection{Noncombatant Discrimination}

One focus of the debates is whether UAVs have the ability to 'better at noncombatant discrimination'. Proponents claim that UAVs are 'better at noncombatant discrimination'. It is an empirical question, namely, it can be verified by the data. Plaw (2013) has calculated UAV attacks carried out by the United States in Pakistan from 2004 to 2007 and concluded that compared to other operations in comparable condition, UAV is able to decrease civilian causality ratio, or at least, not to increase the ratio. The study purportedly provides a powerful empirical evidence to back up the view that UAV is not only ethically permissible but also 'ethically obligatory for military use' (Yoram, 2004).

Admittedly, the fact based on the data is noncontroversial. However, there is the logic fallacy in the inference course, which make their conclusions unsound (International Committee of the Red Cross, 1977).

The first loophole is that proponents mix up UAVs and the operator of UAVs. Current UAVs still need an operator to make a decision, namely, 'human in the loop'. Otherwise, we will come back into the discussion of autonomous weapon. therefore, the precise statement should be that it is the pilots with the help of UAVs, rather than UAVs are "capable of making determinations of proper target discrimination" (Walzer, 2006). What UAVs do is to improve the accuracy of the targeting technically.

The Second loophole is that they fail to distinguish the value from the fact. just as Sarah and John (2012) pointed out, sound ethical decisions based on the distinction between distinguish value and fact (Chris, 2010). If we 
assume that UAV is 'better at noncombatant discrimination', then the assumption implicitly imply that UAV can identify precisely who is noncombatant. However, the definition of noncombatant itself is highly contentious because it involves the judgement of innocent. The nub of the problem is that the judgement of innocence is a value judgement, rather than factual declaration. A factual statement is empirical while value judgement is normative. The former is uncontroversial, such as accounted data about casualties, whereas the latter is full of controversy, such as civilian casualties, because the defining of 'civilian' involves value judgement so that those who hold different moral perceptions will make different judgement to the same individual. therefore, in terms of 'noncombatant discrimination', human beings still have many problems to solve, how we can declare UAVs in the present have a better ability to 'noncombatant discrimination'? Precisely, we agree that UAVs have accurate targeting ability, rather than noncombatant discrimination ability.

\subsection{The Identification of Legal Military Objectives}

Article 52 (2) of the Protocol Additional to the Geneva Convention, defines military objectives as "limited to those objects which by their nature, location, purpose or use make an effective contribution to military action and whose total or partial destruction, capture or neutralization, in the circumstances ruling at the time, offers a definite military advantage." And Article 57 makes a further requirement that strikers must "do everything feasible" to ascertain that the objective should be a legal military objective.

While everyone would agree with the principle, it is difficult to turn the idealistic principle into a practical scheme. It is an acid test for a commander or operator in warfare to verify that directing only those objects that present 'definite military advantage'. We need both humanitarian concerns and a workable operation manual to put it in operation.

The forms of the conflict have experienced great changes, ranging from traditional war to asymmetric conflict, from states actors to non-state actors, from conventional battlefields with straightforward distinction between civilians and combatants to highly controversial civilian-combatant intermingled populated areas, even population centers. All these make it more difficult to make a proper distinction between legal military objectives and illegal objectives. Especially for war on terror, civilians are intermingled with combatants, and many civilian facilities can be transformed into military objectives, let alone those dual-use facilities and technologies. Just as Antonio Cassese comment, the definition of the military objectives is "so sweeping that it can cover practically anything"(Sarah and John, 2012).

\section{The Principle of Proportionality}

Proportionality is the most contentious issue in the moral debate about UAVs, although it is seemingly outlined in the law clearly. The standard of proportionality is delineated in Articles 51(5)(b) and 57(2)( iii) of Additional Protocol I, which proscribe" an attack which may be expected to cause incidental loss of civilian life , injury to civilians, damage to civilian object, or a combination thereof, which would be excessive in relation to the concrete and direct military advantage anticipated" (Braun and Brunstetter, 2013).

According to Waltzer's definition, the principle of proportionality can be concisely described that 'the anticipated collateral damage of an action cannot be excessive in relation to the anticipated military advantage' (US Department of the Army, 2007). It is the proportionality calculation that makes the interpretation and assessment of the principle become the most controversial issue. How to define and measure 'anticipated collateral damage' and 'anticipated military advantage' determine whether the calculating conclusion is 'excessive', which is moral base of 'military necessity'.

Disagreements over the issue are partly a matter of subjective preferences caused by inherent moral concerns, partly a matter of ambiguous concepts. Akin to the problems facing the principle of distinction, what is in issue is not literal meaning, but rather problems of interpretation and feasible solutions.

\subsection{Contentious Proportionality Calculation}

Proportionality calculation is not a simply calculus based on straightforward and unambiguous algorithms, but rather predictive calculations of future possibilities, full of uncertainty. More importantly, it is a tough value-laden ethical decision of attaching weights to the variables at stake. The course of valuating is subjective, influenced by a set of factors, such as circumstances....in addition, it is important to bear in mind the point that proportionality calculation is probabilistic. The calculating determination is highly based on personal experience and evaluation, the accuracy of accessible intelligence. Christian (2017) therefore, it is virtually impossible to "identify a clear, uncontroversial threshold at which anticipated military advantage exceeds anticipated civilian damage" (Joseph, 2017).

A good example to illustrate this issue is the comparison study made by Braun and Brunstetter (2013). Considering the difficulty of delineating the tolerant threshold of damage, they choose collateral damage ratio as a proxy for proportionality to compare the proportionality of CIA's drone strikes and U.S. military's strikes. The result shows that CIA apparently is more tolerant of civilian casualties than the military (Thomas, 2017). Ostensibly, both the military and CIA are under the same umbrella of the proportionality principle, within the same external restraint, decision-making circumstances, decision-making procedure and so on, but CIA and the military adopt divergent threshold respectively. However, the U.S. military and CIA show respectively divergent tolerant thresholds for collateral damage. Probably there's a couple of reasons for the difference. What deserves close consideration is that the influence of strategic ends on proportionality calculation. Considering the role of the subjective factors in the ethical decision, it is a serious problem to avoid the policymakers' temptation to weight the proportionality calculation in favor of the propensity they intend to wage a war. Otherwise, plausible proposition calculation would turn into the means of the belligerent to attempt to justify their behavior of waging war.

\subsection{Anticipated Military Advantage Vs Collateral Damage}

How to calculate military advantage, the US military gives an operational guide. The US Counterinsurgency Field Manual describe that "advantage is best calculated not in terms of how many insurgents are killed or 
detained, but rather which enemies are killed and detained. If certain insurgent leaders are essential to the insurgents' ability to conduct operations, then military leaders need to consider their relative importance when determining how best to pursue them" (Marcus, 2016).

Here the 'relative importance' in the paragraph means that attaching corresponding values to various targets according to the target's value. Admittedly, it is generally accepted that a high-value target deserves a high weighted proportion in the calculation. However, when it comes to try to give a specific value to lives, it would never be an easy work. Specifically, to kill a top-level opposite combatant, how much civilian casualties in risk is acceptable? Besides the target's value, there are a complex of factors should be considered, such as the confidence level of intelligence.

The fulcrum of the requirement is the term "relative importance". If the war goals is open-ended, such as the eradication of evil, the battlefield border would be more open, even unlimited, and a military adversary would be broader, thereby the greater military advantage. It is a temptation for decision makers to weight a proportionality calculation in favor of the warfare they want. Especially without adequate transparency and outsider supervise, it is easier to give a partial evaluation because policymakers are easily influenced by their own subject sentiment preference when evaluating proportionality and converting 'relative importance' into practical proportionality calculating result.

\subsection{Reflections on Debates}

Based on the reflections over debates revolving around the use of UAVs, the paper suggests that there are some deficiencies for further refinement to avoid misleading discussion and better the current moral performance.

\subsubsection{The Percision of the Language}

Admittedly, the ambiguity of the language in the field of ethic is unavoidable. And sometimes ambiguity itself is a kind of strategy, reducing the entry cost to attract more states to ratify treaty or something, which is beneficial to build the way to the consensus of the international community. However, the cost of ambiguity is clearly evident. Ambiguity easily incur disputes, or make the involved party easier to dodge the obligation for its role. More importantly, ambiguity produce meaningless, unconstructive debates which are unhelpful to accumulate wisdom, and even cause wrongly judge.

In addition, interpretations should be more workable and pragmatic. Actually, there is not too much disagreement in principle. Nevertheless, some articles elaborating the principle are either too opacity in text to grasp their inherent meanings precisely or impractical to be achieved, which impede the way to obey the principles in a workable way. Moral debates should not only produce ideas or theories, should also take the responsibilities of working towards a pragmatic solution to moral problems of UAVs in the real world.

\subsubsection{The Transparency}

What is most disputable over the principle of proportionality is the justification of proportionality calculation. Disputes partly arise from the different interpretation of the term, but more stems from the scarce data and extremely opaque decision process. With more transparency, the government would be under more oversight and its action would be more restricted. At the same time, with more transparency the government could persuade the populace to believe in their accountability and their justification of their waged strikes in either traditional war or war on terror.

\section{Conclusion}

In conclusion, we tend to agree that the hope to ban thoroughly is unrealistic. There are too many handles on the way. The first reason is that neither in the technological field nor policy circle has common sense about the issue. The second reason is that interests. Now a hard-bounding treaty has not existed. Due to the incremental character of the technological advancement from automation to autonomy, there are many uncertainties in development which are beyond the human cognition in the current phrase. Therefore, defining the key term precisely is an impossible fulfilled work. The only solution is that substitute precise statement by an ambiguous statement and the subsequent consequence is that ambiguity arouses confusing, conflating and so on, just like the awkward situation discussed in the above context. Even the treaty has come into being, it would be difficult to persuade parties with respective interests to ratify a treaty with effective bounding power. Substantial military advantages will constitute a prisoner dilemma.

Based on the view, we contend that ban is unfeasible. Compared to banning, it is more feasible to keep constant vigilance, make incremental modification according to continuous changing. Specifically, in the fields of language precision and information transparency, adjust cognition and adapt to changes.

\section{References}

Braun, M. and D.R. Brunstetter, 2013. Rethinking the criterion for assessing CIA-targeted killings: Drones, proportionality and jus ad vim. Journal of Military Ethics, 12(4): 304-324.Available at: https://doi.org/10.1080/15027570.2013.869390.

Chris, J., 2010. Law from above: Unmanned aerial systems, use of force, and the law of Armed conflict. North Dakota Law Review, 85: 649671.

Christian, E., 2017. Drones, risk, and moral injury. Critical Military Studies: 1-18.Available at: 10.1080/233374886.2017.1384979.

International Committee of the Red Cross, 1977. Protocols additional to the Geneva Conventions of 12 August 1949: Resolutions of the Diplomatic Conference: Extracts from the Final Act of the Diplomatic Conference. International Committee of the Red Cross.

Joseph, C.O., 2017. Remotely piloted aircraft, risk, and killing as sacrifice: The cost of remote warfare. Journal of Military Ethics, 16(3-4): 256-271.Available at: https://doi.org/10.1080/15027570.2018.1440501.

Kiggins, R., 2018. Robots and political economy. In the political economy of robots. Cham: Palgrave Macmillan. pp: 1-16.

Lee, P., 2018. Armed drones: Automation, autonomy, and ethical decision-making. In the political economy of robots. Cham: Palgrave Macmillan. pp: 291-315.

Marcus, S., 2016. Rethinking military virtue ethics in an age of unmanned weapons. Journal of Military Ethics, 15(3): 187-204.Available at: https://doi.org/10.1080/15027570.2016.1257851. 
MoD, U.K., 2011. Joint doctrine note 2/ 11 the UK approach to unmanned aircraft systems. Swindon, Wiltshire: UK MoD The Development, Concepts and Doctrine Centre, 30 .

Plaw, A., 2013. Counting the dead: The proportionality of predation in Pakistan. Killing by Remote Control: The Ethics of an Unmanned Military: 126-153.

Sarah, K. and K. John, 2012. The use of unmanned aerial vehicles in contemporary conflict: A legal and ethical analysis. Polity, 44(2): 260285.Available at: https://doi.org/10.1057/pol.2012.2.

Strawser, B.J., 2010. Moral predators: The duty to employ uninhabited aerial vehicles. Journal of Military Ethics, 9(4): 342-368.Available at: https://doi.org/10.1080/15027570.2010.536403.

Thomas, G., 2017. Targeted killings: Drones, noncombatant immunity, and the politics of killing. Contemporary Security Policy, 38(2): $212-$ 236.Available at: https://doi.org/10.1080/13523260.2017.1336296.

US Department of the Army, 2007. Counterinsurgency field manual, FM 3-24, MCWP 3-33.5. London: University of Chicago Press. pp: 721.

Walzer, M., 2006. Just and unjust wars: A moral argument with historical illustrations. 4th Edn., New York: Basic Books.

Yoram, D., 2004. The conduct of hostilities under the law of international Armed conflict. Cambridge; New York: Cambridge University Press. 